\title{
Intraperitoneal administration of butyrate prevents the severity of acetic acid colitis in rats
}

\author{
Joshua J. MALAGO ${ }^{\dagger 1}$, Catherine L. SANGU ${ }^{2}$ \\ ( ${ }^{1}$ Department of Pathology, Faculty of Veterinary Medicine, Sokoine University of Agriculture, P.O. Box 3203, Morogoro, Tanzania) \\ ( ${ }^{2}$ c/o Walter Oseko, P.O. Box 62, Duluti, Arusha, Tanzania) \\ ${ }^{\dagger}$ E-mail: malagojj@yahoo.com
}

Received July 7, 2014; Revision accepted Dec. 3, 2014; Crosschecked Feb. 17, 2015

\begin{abstract}
Intrarectal infusion of butyrate improves colorectal disorders including ulcerative colitis (UC). However, it is not established whether systemically administered butyrate benefits such patients. The current study aimed at exploring and comparing the potential of intraperitoneally, intrarectally, and orally administered butyrate against acetic acid (AA)-induced UC in rats. Intrarectal administration of $2 \mathrm{ml}$ of $50 \%$ AA was done after or without prior treatment of rats for 7 consecutive days with $100 \mathrm{mg} / \mathrm{kg}$ sodium butyrate (SB) intraperitoneally, intrarectally, or orally. Rats were sacrificed after $48 \mathrm{~h}$ of AA-treatment. Subsequently, colon sections were processed routinely for histopathological examination. We clinically observed diarrhea, loose stools, and hemoccult-positive stools, and histologically, epithelial loss and ulceration, crypt damage, goblet cell depletion, hemorrhage, and mucosal infiltration of inflammatory cells. The changes were significantly reduced by intraperitoneal, intrarectal, or oral butyrate, with intraperitoneal butyrate exhibiting the highest potency. It is concluded that intraperitoneal administration of butyrate abrogates the lesions of AA-induced UC and its potency surpasses that of intrarectal or oral butyrate.
\end{abstract}

Key words: Butyrate, Oral administration, Intraperitoneal administration, Intrarectal administration, Acetic acid, Ulcerative colitis

doi: $10.1631 /$ jzus.B1400191

Document code: A

CLC number: R574.62

\section{Introduction}

Ulcerative colitis (UC) is a form of inflammatory bowel disease (IBD) whose exact etiology remains enigmatic. Perturbations of the immune system along the gut are implicated in playing a central role. In its active form, UC is characterised by constant diarrhea mixed with blood of gradual onset and weight loss. Blood can also be seen on rectal examination and varying degrees of abdominal pain ranging from mild discomfort to painful bowel movements and/or abdominal cramping during bowel movements may accompany the disease (Torpy et al., 2012). Morphologically, UC is mainly characterized by open sores or ulcers in the colon, loss of epithelium and

(ㄹ) ORCID: Joshua J. MALAGO, http://orcid.org/0000-0002-8903-210X (C) Zhejiang University and Springer-Verlag Berlin Heidelberg 2015 goblet cells, and infiltration of colonic mucosa by inflammatory cells (Malago and Nondoli, 2008).

Although the exact cause of UC is unknown, it is suggested that over stimulation or inadequate regulation of the mucosal immune system is a major pathophysiologic cause of the disease. Continual activation of the immune system to produce proinflammatory chemokines like interleukin (IL)-8 and their subsequent attraction of inflammatory cells that infiltrate the colon mucosa are cascade events that cause the chronic inflammation and ulceration of the colonic epithelium (Malago and Nondoli, 2008; Ke et al., 2013; Liu et al., 2013). The production of these pro-inflammatory cytokines is mediated mainly by transcriptional activation of nuclear factor $\kappa B$ (NF- $\mathrm{\kappa B}$ ) and mitogen activated protein kinase (MAPK) pathways (Malago et al., 2002; Ke et al., 2013). Several therapeutic interventions such as butyrate enemas 
modulate the activation of these pathways and suppress the production of pro-inflammatory cytokines to protect the colon against inflammatory injury (Venkatraman et al., 2003; Malago et al., 2005; Ke et al., 2013).

Earlier studies that linked the development of UC and butyrate levels in the colon, observed that deficiency of butyrate leads to disease development and that restoration of butyrate levels by intracolonic infusion treats UC (Roediger, 1980). Since then, butyrate enemas have popularly been used as medicaments stemming from their potential to impart beneficial attributes to the colon. This potential involves an increase in mechanical strength of injured colonic mucosa to hasten the healing process (Bloemen et al., 2010; Mathew et al., 2010), suppression of IL-8 production by intestinal epithelial cells to protect against the inflammatory process (Malago et al., 2005), and clinical remission of UC by protecting against inflammatory and oxidative stress parameters of the disease (Hamer et al., 2010b). Much as butyrate tends to impart a protective effect, several authors have indicated failures or limited success of butyrate to relieve IBD patients (Harig et al., 1989; Sanderson, 1997; Hamer et al., 2010b). One of the proposed reasons includes presence of IL-8 in the intestinal mucosa, which is either activated (Fusunyan et al., 1998; 1999) or inhibited (Huang et al., 1997; Wu et al., 1999) by different doses of butyrate (Malago et al., 2005). Thus high concentrations of butyrate correlate with elevated IL-8 levels in the intestinal mucosa of IBD patients (Dabard et al., 1987; Treem et al., 1994) whereas low levels diminish the disease activity (O'Morain et al., 1984). As the intestinal concentration of butyrate is mainly determined by diet and commensal microbial profile which varies among individuals, the effect of butyrate on chronic IBD remains controversial.

To impart its attributes, butyrate needs to be absorbed by the colonocytes and undergo the subsequent metabolism. Butyrate absorption mainly occurs in the proximal colon whose function is impaired during UC due to damaged epithelial mucosa (Thibault et al., 2010). As a result, there is impairment of butyrate metabolism even when the colon is saturated with butyrate (de Preter et al., 2011; 2012; Kovarik et al., 2011). Furthermore, addition of compounds interfering with the $\beta$-oxidation pathway such as carnitine has no effect on butyrate metabolism in active UC (de Preter et al., 2011) and has little effect in mild-to-moderate UC following oral intake (Mikhailova et al., 2011). These observations suggest that absorption of intrarectally administered butyrate can be impaired in active UC and may significantly lower the expected beneficial effects of butyrate. Instead, UC patients could most probably benefit more when butyrate reaches the colon through a systemic route. Unfortunately the effects of parenteral routes of high absorptive properties to the benefit of butyrate in UC have not been studied. In this study we explored the differences in the effect of oral, intraperitoneal, and intrarectal administration of butyrate on a rat model of acetic acid (AA)-induced UC.

\section{Materials and methods}

\subsection{Animals}

The experiment used 40 male Wistar rats (Sokoine University of Agriculture, Morogoro, Tanzania) aged 26 weeks and weighing 110 to $130 \mathrm{~g}$. The animals were kept in a restricted access room in which the temperature was controlled and a 12-h light/dark cycle was observed. They were randomly kept in 10 cages (serving as treatment groups) each containing 4 animals (Table 1). The animals were allowed 2 weeks to acclimatize in their groups prior to commencement of the experiment. They were fed standard laboratory diet and given drinking water ad libitum. The study was approved by the Animal Research Committee of the Sokoine University of Agriculture, Tanzania.

Table 1 Animal groups and treatments

\begin{tabular}{|c|c|c|c|}
\hline \multirow{2}{*}{ Group } & \multirow{2}{*}{ Treatment } & \multicolumn{2}{|l|}{ Dose } \\
\hline & & Days $1-7$ & Day 8 \\
\hline 1 & SB & SB: $2 \mathrm{ml}, 100 \mathrm{mg} / \mathrm{kg}$, OS & NS: $2 \mathrm{ml}, \mathrm{IR}$ \\
\hline 2 & SB & SB: $0.2 \mathrm{ml}, 100 \mathrm{mg} / \mathrm{kg}$, IP & NS: $2 \mathrm{ml}, \mathrm{IR}$ \\
\hline 3 & SB & SB: $0.2 \mathrm{ml}, 100 \mathrm{mg} / \mathrm{kg}$, IR & NS: $2 \mathrm{ml}, \mathrm{IR}$ \\
\hline 4 & $\mathrm{SB}+\mathrm{AA}$ & $\mathrm{SB}: 2 \mathrm{ml}, 100 \mathrm{mg} / \mathrm{kg}$, OS & AA: $2 \mathrm{ml}, \mathrm{IR}$ \\
\hline 5 & $\mathrm{SB}+\mathrm{AA}$ & SB: $0.2 \mathrm{ml}, 100 \mathrm{mg} / \mathrm{kg}$, IP & AA: $2 \mathrm{ml}, \mathrm{IR}$ \\
\hline 6 & $\mathrm{SB}+\mathrm{AA}$ & SB: $0.2 \mathrm{ml}, 100 \mathrm{mg} / \mathrm{kg}, \mathrm{IR}$ & AA: $2 \mathrm{ml}, \mathrm{IR}$ \\
\hline 7 & AA & NS: $0.2 \mathrm{ml}$, IR & AA: $2 \mathrm{ml}, \mathrm{IR}$ \\
\hline 8 & Control & $\mathrm{NS}: 2 \mathrm{ml}, \mathrm{OS}$ & NS: $2 \mathrm{ml}, \mathrm{IR}$ \\
\hline 9 & Control & NS: $0.2 \mathrm{ml}$, IP & NS: $2 \mathrm{ml}, \mathrm{IR}$ \\
\hline 10 & Control & $\mathrm{NS}: 0.2 \mathrm{ml}, \mathrm{IR}$ & NS: $2 \mathrm{ml}$, IR \\
\hline
\end{tabular}

All animals were sacrificed on Day 10. SB: sodium butyrate; AA: acetic acid; NS: normal saline; OS: per oral; IP: intraperitoneal; IR: intrarectal 


\subsection{Administration of AA to induce UC}

In order to induce UC, we intrarectally administered $2 \mathrm{ml}$ of $4 \%$ (v/v) AA (Rankem, New Delhi, India) using a soft, $1.5 \mathrm{~mm}$ diameter butterfly catheter (Neomedic Limited, 97 High Street, Rickmansworth, Hertfordshire, UK). The catheter was inserted into the anus as far as the proximal colon (about $8 \mathrm{~cm}$ long), and then AA was released slowly to ensure that it was in contact with the mucosa for sufficient time. After the chemical was administered, the catheter was withdrawn slowly to avoid any physical trauma to the intestinal mucosa.

\subsection{Effect of butyrate on AA colitis}

To explore the effect of butyrate on AA-induced UC, sodium butyrate (SB) (Merck Schuchardt OHG, Hohenbrunn, Germany) was given as indicated in Table 1. Briefly, SB was given at $100 \mathrm{mg} / \mathrm{kg}$ body weight orally, intraperitoneally, or intrarectally once a day for 7 consecutive days. On Day 8, rats received AA to develop UC for $48 \mathrm{~h}$, during which clinical signs were observed. On Day 10 all animals were sacrificed humanely by chloroform. We provided either SB or physiological saline to matched control groups at a similar route, volume, dose, and time.

\subsection{Clinical evaluation of UC}

In order to clinically assess AA colitis activity, we examined the rats for stool consistency which included loose stool and diarrhea, occult and/or bleeding which included hemoccult positivity and gross bleeding, and body weight. We expressed the body weight as percentage weight change for each individual rat. It was calculated in comparison to that of Day 8 before administration of AA. From these data, a disease activity index was calculated as done previously (Malago and Nondoli, 2008) (Table 2).

\subsection{Evaluation of histological changes}

Midline laparotomy was done to open the sacrificed rats. Accessed colon was longitudinally opened,
Table 2 Scoring of AA-induced colitis activity index

\begin{tabular}{cccc}
\hline Score & $\begin{array}{c}\text { Weight } \\
\text { loss }(\%)\end{array}$ & $\begin{array}{c}\text { Stool } \\
\text { consistency }\end{array}$ & $\begin{array}{c}\text { Occult/gross } \\
\text { bleeding }\end{array}$ \\
\hline 0 & None & Normal & Normal \\
1 & $1-5$ & & \\
2 & $5-10$ & Loose stool & Hemoccult positive \\
3 & $10-20$ & & \\
4 & $>20 \%$ & Diarrhea & Gross bleeding \\
\hline
\end{tabular}

The AA colitis activity index was obtained by combining the scores of weight loss (calculated to the day of, but before, AA administration), stool consistency, and bleeding divided by $3 .{ }^{1}$ Normal stools: well formed pellets; Loose stools: pasty and semi-formed stools, which do not stick to the anus; Diarrhea: liquid stools that stick to the anus

fixed with $10 \%(0.1 \mathrm{~g} / \mathrm{ml})$ neutral buffered formalin, and embedded in paraffin. It was then sectioned at $4 \mu \mathrm{m}$ slices that were deparaffinised on microscopic glass slides before staining with hematoxylin and eosin (H\&E). After mounting, the slides were observed under a light microscope (Olympus BX41, Olympus, Japan). Two independent pathologists assessed and quantitated in a blind manner the mucosal integrity for histological evaluation of tissue damage as done previously (Malago and Nondoli, 2008). The evaluation was done on distal colon using a 0-3 scoring scale as shown in Table 3.

\subsection{Statistical analysis}

Numerical values were calculated and presented as mean \pm standard error of the mean (SEM). One-way analysis of variance (ANOVA) was used to assess statistical significance between the mean values of control and treated rats with comparison of means. Differences were considered significant at $95 \%$ confidence interval using Student's $t$-test.

\section{Results}

\subsection{Clinical AA colitis and effect of butyrate}

Fig. 1 shows the clinical signs of AA colitis in rats with or without prior treatment with butyrate. As shown in this figure, AA induced hemoccult-positive

Table 3 Histological score of colitis

\begin{tabular}{ccccc}
\hline Score & Loss of epithelium (\%) & Crypt damage $^{1}(\%)$ & Depletion of goblet cells & Infiltration of inflammatory cells \\
\hline 0 & None & None & None & None \\
1 & $<5$ (mild) & $<10$ (mild) & Mild & Mild \\
2 & $5-10$ (moderate) & $10-20$ (moderate) & Moderate & Moderate \\
3 & $>10$ (severe) & $>20$ (severe) & Severe & Severe \\
\hline
\end{tabular}

\footnotetext{
${ }^{1}$ Crypt damage was evaluated as percentage loss of crypt
} 
stool, diarrhea, and loose stool. The hemoccultpositive stool was the severest sign observed in one third $(33 \%)$ of the animals. This was followed by diarrhea in $50 \%$ of AA colitis animals. The least severe sign was loose stool encountered in $17 \%$ of these animals. None of the animals receiving AA had a normal stool.

Butyrate effect on AA colitis was exhibited by improvement of colitis signs in a route-dependent manner. According to Fig. 1, all tested butyrate routes (oral, intraperitoneal, and intrarectal) ameliorated the hemoccult-positive stools in all animals. It is apparent from this figure that the potency of butyrate to suppress the severity of AA colitis was routedependent. Oral butyrate was the least potent as it had a high number of animals (75\%) with diarrhea and few $(25 \%)$ with loose stools. This potency increased with intrarectal butyrate where diarrhea was reduced further by $50 \%$, leading to $75 \%$ of animals showing loose stools and only $25 \%$ having diarrhea. The strongest butyrate effect was observed in animals receiving intraperitoneal butyrate. In this case, diarrhea had disappeared, $25 \%$ of animals showed loose stools, and the majority $(75 \%)$ recovered from AA colitis signs as manifested by normal stools. Rats receiving butyrate alone or normal saline in any of the three routes did not show any of the clinical signs and had normal stools.

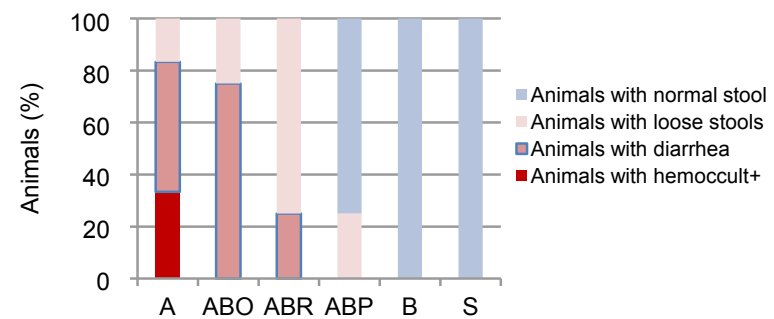

Fig. 1 Effect of sodium butyrate on clinical indices of colitis

Rats were treated intrarectally with $4 \%$ acetic acid alone (A) or after 7 consecutive days of treatment with $100 \mathrm{mg} / \mathrm{kg}$ sodium butyrate orally (ABO), intrarectally (ABR), or intraperitoneally (ABP). Values for control rats receiving butyrate alone (B) or physiological saline (S) are also indicated. Results are expressed as the percentage of animals. Eight animals were used for each experimental group

\subsection{AA colitis activity index}

Fig. 2 indicates that AA induced a disease activity index of about 3 . This activity was reduced significantly by all routes of butyrate administration.
Oral or intrarectal butyrate reduced the AA activity index about 2.5 -fold whereas intraperitoneal butyrate reduced it 5-fold. Comparison among butyrate administration routes indicated a significant difference between intraperitoneal butyrate and either oral or intrarectal butyrate on reducing the AA activity index. In that case, intraperitoneal butyrate reduced the disease activity by 2 -fold more than either oral or intrarectal butyrate. No disease activity was observed in animals receiving butyrate or normal saline alone.

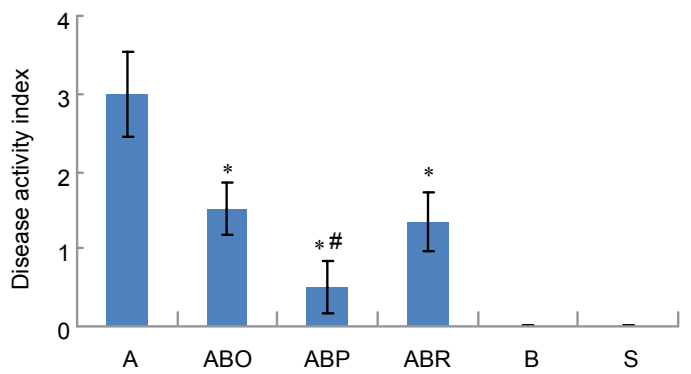

Fig. 2 Effect of sodium butyrate administration on acetic acid (AA) colitis disease activity index

Rats were treated intrarectally with $4 \%$ acetic acid alone (A) or after 7 consecutive days of treatment with $100 \mathrm{mg} / \mathrm{kg}$ sodium butyrate orally $(\mathrm{ABO})$, intrarectally (ABR) or intraperitoneally (ABP). Values for control rats receiving butyrate alone (B) or physiological saline (S) are also indicated. Disease activity index is obtained by combining scores of weight loss, stool consistency, and bleeding divided by 3 . Results are expressed as mean \pm SEM $(n=8)$. * Significantly different from AA colitis group at $P<0.05$. ${ }^{\#}$ Significantly different from another route of butyrate administration at $P<0.05$

\subsection{Gross pathology}

Gross lesions of colon mucosa from rats treated with AA were edematous with hemorrhagic erosions scattered throughout the colon. In some animals, less severe lesions with edema of the colon, multiple mucosal erosions and marked congestion of mesenteric blood vessels were observed. The lesions were more severe in the distal colon than in the middle and proximal colons. Rats treated with butyrate orally or intrarectally prior to AA had less congestion of the colon without edema. Those given butyrate intraperitoneally had mild congestion and some appeared normal. The butyrate-treated and control rats had no gross pathological lesions (data not shown).

\subsection{Histopathological findings}

The observed histopathological lesions due to AA following or without prior treatment with SB are 
shown in Fig. 3. According to Fig. 3a, the histological changes due to AA were vivid. They included massive haemorrhage, complete absence of epithelial lining on the mucosal surface, severely damaged villi and crypts with subsequent loss of their architecture except in a few crypts, depletion of goblet cells, and severe infiltration of inflammatory cells. Compared with rats receiving butyrate prior to AA (Figs. 3b-3e), these rats were the most severely affected.

Figs. 3b-3e show the effect of prior treatment of rats with butyrate on AA-induced UC. Rats receiving butyrate intrarectally had a considerably distorted but intact epithelial lining on the mucosal surface underneath which there was haemorrhage that occupied mainly the intervillous and intercryptal spaces. The villi were moderately damaged, their architecture could be traced with difficulty in some areas, and most of their goblet cells were lost. There was mild damage to the crypts and retention of most of their goblet cells. In addition, there was a vivid infiltration of inflammatory cells in the mucosa. There was little difference between these animals and those exposed to AA after oral butyrate. In the latter, villous damage was mild, the architecture of most villi was easily traceable and the loss of goblet cells was mild. Although the histology of the colon remained distorted in both treatments, when compared to sections of rats exposed to AA alone (Fig. 3a), it is obvious that intrarectal (Fig. 3d) or oral (Fig. 3b) butyrate significantly benefited rats against the AA-induced epithelial damage.

Rats receiving butyrate intraperitoneally prior to AA were least severely affected when compared to those given intrarectally or orally. Their colon histology looked almost normal with quite a good epithelial lining, intact villi and crypts, undamaged goblet cells, mild haemorrhage, and little mucosa infiltration by inflammatory cells (Fig. 3c). Indeed, these rats had highly improved from the UC lesions when compared to those not receiving butyrate (Fig. 3a).

The histological results of rats exposed to butyrate alone are shown in Fig. 3f. According to this figure, the epithelial lining is intact, the crypts are not damaged, goblet cell depletion is lacking or very low, and there is very mild inflammatory cells infiltrating the mucosa. These results are more or less the same as those from the normal control animals receiving normal saline (data not shown).

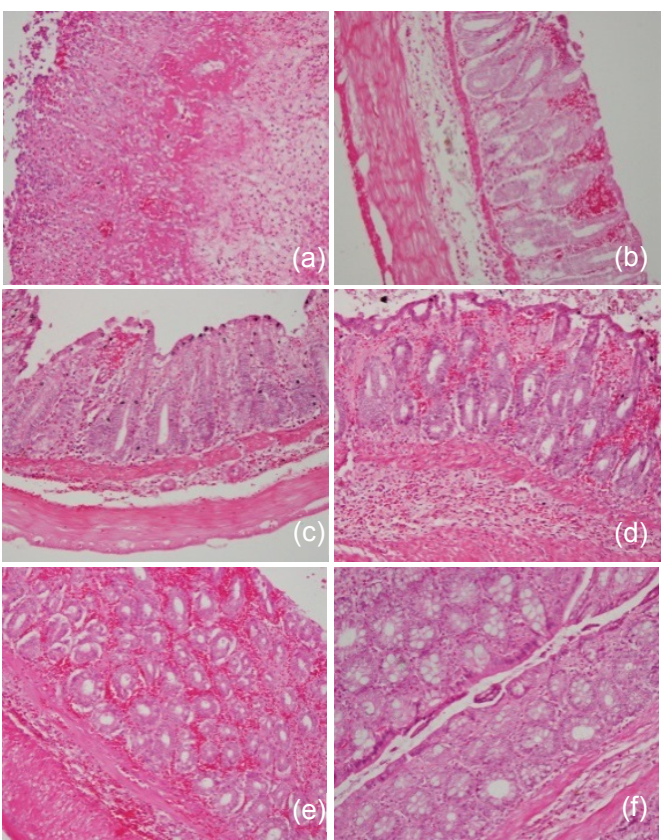

Fig. 3 Histological sections of rat colon

Rats were treated intrarectally with $4 \%$ acetic acid alone (a), or following $100 \mathrm{mg} / \mathrm{kg}$ sodium butyrate orally (b), intraperitoneally (c), or intrarectally (d and e). (a) Massive hemorrhage, loss of epithelial lining, loss of crypt and villi, and massive infiltration of inflammatory cells due to acetic acid; (b) Considerable intact epithelium under which there is moderate hemorrhage between crypts and villi, loss of some goblet cells, and moderate infiltration of inflammatory cells in the submucosa; (c) A more intact epithelium, mild hemorrhage, and mild infiltration of inflammatory cells; (d) A considerable intact epithelium with severe hemorrhage underneath and destroyed villi and some crypts, and severe loss of goblet cells in villi, severe cellular infiltration in the submucosa; (e) Intercryptal hemorrhage and retention of crypt architecture; (f) The photomicrographs also include those of control rats treated with sodium butyrate alone with no significant changes. Each photo represents 8 animals (4 animals per treatment in duplicate experiments). Hematoxylin and eosin, original magnification $\times 200$

\subsection{Scores of histopathological lesions}

Fig. 4 presents scores of histopathological lesions. It is clear that exposure to AA led to a severe (score 3) loss of epithelium (Fig. 4a), crypt damage (Fig. 4b), depletion of goblet cells (Fig. 4c), and infiltration of inflammatory cells (Fig. 4d). Pre-treatment with butyrate significantly reduced the severity of these changes. The reduction of epithelial loss was to about half following oral or intrarectal butyrate and to almost complete abrogation when butyrate was given intraperitoneally (Fig. 4a). Crypt damage was reduced by one third (score 2, moderate damage), two thirds (score 1, mild damage), or complete abrogation (score 
(a)

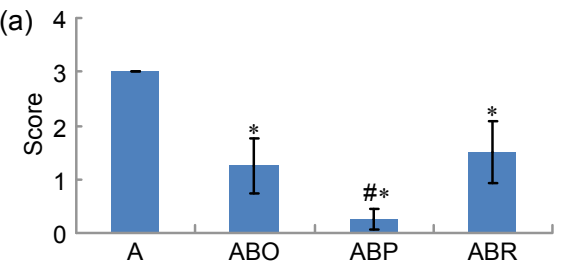

(c)

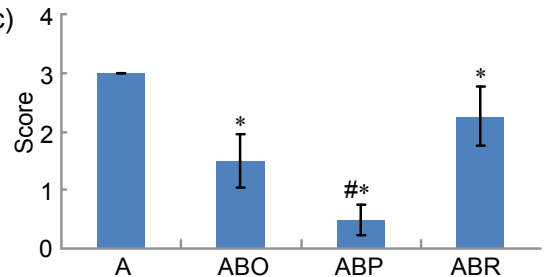

(b)

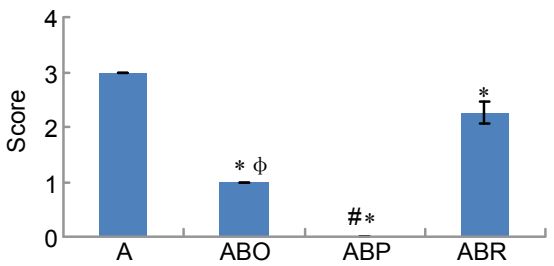

(d)

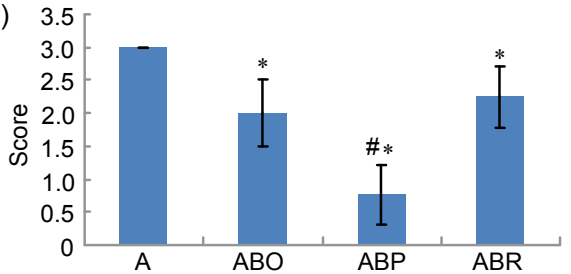

Fig. 4 Histological scores of loss of epithelium (a), crypt damage (b), depletion of goblet cells (c), and infiltration of inflammatory cells $(d)$

Rats were treated intrarectally with $4 \%$ acetic acid (AA) alone (A) or after 7 consecutive days of treatment with $100 \mathrm{mg} / \mathrm{kg}$ sodium butyrate orally (ABO), intrarectally (ABR), or intraperitoneally (ABP). Results are expressed as mean $\pm \mathrm{SEM}$ of at least 8 individual rats. " Significantly different from AA exposed rats $(P<0.05) ;{ }^{\#}$ Significantly different from another routes of butyrate administration at $P<0.05 ;{ }^{\Phi}$ Significantly different between oral and intrarectal butyrate at $P<0.05$

0 , no damage) when butyrate was given intrarectally, orally, or intraperitoneally, respectively (Fig. 4b). The preventive effect of butyrate on goblet cell depletion varied from about 1-fold (moderate depletion) with intrarectal administration through 2-fold (moderate depletion) with oral butyrate to 6-fold (mild depletion) after intraperitoneal butyrate (Fig. 4c). Fig. 4d indicates that butyrate reduced the severe infiltration of inflammatory cells to moderate (score 2) when given orally or intrarectally and to mild (score 1) by intraperitoneal butyrate.

Fig. 4 further indicates that the effects of intraperitoneal butyrate in preventing epithelial loss (Fig. 4a), crypt damage (Fig. 4b), depletion of goblet cells (Fig. 4c), and infiltration of inflammatory cells (Fig. 4d) were significantly different from those of oral or intrarectal administration of butyrate. When the effects of oral and intrarectal butyrate were compared, oral administration of butyrate tended to have a higher suppressive effect to these lesions, which was significant only for crypt damage (Fig. 4b). Butyrate alone did not cause any significant changes in the epithelium, crypt, goblet cells or infiltration of inflammatory cells into the colon mucosa.

\section{Discussion}

Our results on the AA-induced UC rat model have harmonized with those of other researchers.
Previous studies have shown that intrarectal administration of AA to create a rat model of $\mathrm{UC}$ results in inflammation and ulceration in the lining of the colon. This can be seen histologically as distortion of crypt architecture, loss of epithelial cells, ulceration, haemorrhage, and neutrophil infiltration (Sotnikova et al., 2013; Minaiyan et al., 2014). In such a condition, the colon cannot absorb liquid from the stools, resulting in a larger volume of watery stools and subsequent weight loss (Zhao et al., 2014). The hemorrhagic and ulcerative colon mucosa may also bleed to produce bloody faeces (Sotnikova et al., 2013). The rat model of AA was thoroughly studied and established to correlate with human UC by several scientists including Fabia et al. (1992) who explored the effect of $4 \%, 6 \%$, and $8 \%$ AA to the rat colon. They found that exposure of the colon to $4 \%$ AA for $15 \mathrm{~s}$ produced a uniform colitis in all treated rats with morphological similarities to human UC. Subsequent studies have reproduced this model with close resemblance to human UC in terms of pathogenesis, histopathological features and inflammatory mediator profile. Consistent noteworthy features of human colitis observed in these models include mucosal hemorrhage, loss of epithelial cells and ulceration, damage of crypts and distortion of crypt architecture, depletion of goblet cells, infiltration of inflammatory cells, particularly neutrophils in lamina propria, necrosis of mucosal and submucosal layers, vascular dilation, and edematous submucosa (La et al., 
2003; Cetinkaya et al., 2005; Randhawa et al., 2014). Indeed, the morphological changes observed in our study were consistent with previous findings in rat models of colitis, which correlate well with human UC.

Topical administration of butyrate to cure colitis has been fairly well demonstrated (Scheppach et al., 1992; Hamer et al., 2010a; 2010b). This is done mainly through intrarectal administration of enemas that contain butyrate. The procedure is one of the earliest approaches to treat UC even in patients who had been unresponsive to or intolerant of standard therapy (Scheppach et al., 1992). The intrarectally administered butyrate needs to be absorbed before it works. Normally butyrate absorption mainly occurs in proximal colon whose function is impaired during UC. This hinders absorption of topically administered butyrate and may not benefit UC patients. However, butyrate absorption in the colon can be increased by manipulating electrolyte composition in the rectal lumen (Holtug et al., 1995) since rectal butyrate absorption remains normal during UC (Hove et al., 1995). Thus, topical butyrate, given intrarectally in form of SB, plays a double role; firstly by employing sodium ions, it accelerates rectal absorption of SB and secondly, the absorbed butyrate imparts healing to the colonocytes. The end result is epithelial proliferation to restore the damaged epithelium, especially the lost colonic epithelial continuity.

Contrary to the obviously evident successful use of topically administered butyrate enemas against UC, studies using the oral route are rare in literature (Vieira et al., 2012) and those for intraperitoneal route are not found. Oral administration of butyrate has been shown to significantly improve trophism and reduce leukocyte infiltration in the colon mucosa and revert to normal the UC-associated alterations of transforming growth factor- $\beta$ (TGF- $\beta$ ), IL-10, T lymphocytes and dendritic cells (Vieira et al., 2012). In doing so, oral butyrate attenuates the inflammatory profile of colonic mucosa. The effect of oral butyrate on UC has further been studied in combination with standard drugs. A randomized, double-blind, placebo-controlled study by Vernia et al. (2000) demonstrated that a combined oral SB and mesalazine treatment results in a more favourable UC trend compared to oral mesalazine alone. The trend includes decreased disease activity index as well as endoscopic and histologic scores. Apart from oral intake of butyrate, other studies have demonstrated that oral administration of dietary fibers that are subsequently fermented in the colon to produce butyrate is as effective as standard therapeutic drugs like mesalamine in maintaining remission of UC (Fernández-Bañares et al., 1999). In addition, several recent studies focusing on the role of orally given butyrate producing bacteria like Butyricicoccus spp. or Clostridium tyrobutyricum, have revealed that the bacteria increase the butyrate levels in the colon and subsequently cure UC (Hudcovic et al., 2012; Eeckhaut et al., 2013). All these facts and our own results in this study consistently indicate that orally given butyrate could be a potential route to treat UC.

We have demonstrated the potential of intraperitoneally administered butyrate to prevent the severity of AA-induced UC lesions. To the best of our knowledge, this finding has not been reported before. However, the systemic effect of butyrate to other body systems and organs has been reported. For instance, intraperitoneal injection of butyrate at 50$200 \mathrm{mg} / \mathrm{kg}$ body weight decreases gentamicininduced nephrotoxicity in rats by enhancing renal antioxidant enzyme activity and expression of prohibitin protein (Sun et al., 2013). When given at $1200 \mathrm{mg} / \mathrm{kg}$, intraperitoneal butyrate ameliorates an aging-associated deficit in object recognition memory in rats (Reolon et al., 2011). Silingardi et al. (2010) further demonstrated that chronic intraperitoneal administration of butyrate to long-term monocularly deprived adult rats causes a complete recovery of visual acuity. A more recent study has also reported that intraperitoneal injections of butyrate for $28 \mathrm{~d}$ to adult $\mathrm{C} 57 \mathrm{BL} / 6$ mice prevent repressed contextual fear memory caused by isoflurane (Zhong et al., 2014). All these facts and our own study affirm that butyrate has a potential to impart protective roles to various body organs and systems through systemic administration.

We have also assessed the AA-induced UC lesions in two other different orders; firstly, AA was given every $24 \mathrm{~h}$ for $2 \mathrm{~d}$ followed by SB for 4 consecutive days and secondly, alternating $\mathrm{AA}$ and $\mathrm{SB}$ each given every fortnight for $6 \mathrm{~d}$. Our results (data not shown) indicate that butyrate given after AA has less protective potency than when given prior to AA and that butyrate does not benefit rats when alternated with AA. Thus in this very study, we extend further the protective potency of systemic butyrate to include 
UC. We chose a $100 \mathrm{mg} / \mathrm{kg}$ dose on the grounds that previous studies using 50,100 , and $200 \mathrm{mg} / \mathrm{kg}$ in rats (Sun et al., 2013) and 100 to $10000 \mathrm{mg} / \mathrm{kg}$ in mice (Ferrante et al., 2003) did not have any detrimental effect. The used dose may also have no effect in humans since intravenous SB dose of $500 \mathrm{mg} /(\mathrm{kg} \cdot \mathrm{d})$ for 10 consecutive days has shown no toxicity in these subjects (Miller et al., 1987). Nonetheless, establishment of the exact amount of butyrate for intraperitoneal administration against human UC needs to be researched.

Although there is no single study that has established the amount of SB reaching the colonocytes following intraperitoneal administration, it is known from human studies that the normal colon butyrate levels of $(5.0 \pm 4.2)$ to $(14.7 \pm 2.9) \mathrm{mmol} / \mathrm{kg}$ chyme correspond to $29 \mu \mathrm{mol} / \mathrm{L}$ of portal blood (Cummings et al., 1987). Furthermore, in vitro studies have indicated that butyrate consumption by colonocytes is 1.5 and $0.5 \mathrm{mmol} / \mathrm{L}$ after 4 and $12 \mathrm{~h}$ of treatment, respectively, even if the high dose of $50 \mathrm{mmol} / \mathrm{L}$ is supplied (Sauer et al., 2007). The $545 \mathrm{mmol} / \mathrm{L}$ solution (calculated from $100 \mathrm{mg} / \mathrm{kg}$ dose, $120 \mathrm{~g}$ average body weight, $0.2 \mathrm{ml} \mathrm{SB}(110.09 \mathrm{~g} / \mathrm{mol})$ per animal) used in our study was far higher than the blood butyrate levels of $29 \mu \mathrm{mol} / \mathrm{L}$. We assumed that our $100 \mathrm{mg} / \mathrm{kg}$ dose will supply enough butyrate to reach the colonocytes at adequate physiological levels despite the fact that butyrate is rapidly used after its absorption in blood. Since diffusion of intraperitoneally injected substances between the fluid in the peritoneal cavity and the blood is limited after reaching equilibrium and the rate of absorption depends on diffusion rate of the substance (Clark, 1921), we suggest that the used SB dose was enough to provide colonocytes a physiological concentration of butyrate for adequate time to impart its effect.

During UC, the inflamed intestinal mucosa impairs butyrate metabolism and predisposes colonocytes to intracellular butyrate deficiency. The impaired butyrate metabolism could be due to luminal compounds interfering with butyrate metabolism, changes in luminal butyrate concentrations or $\mathrm{pH}$, and defects in $\beta$-oxidation or butyrate transport (Thibault et al., 2010). Butyrate deficiency develops from decreased uptake of butyrate by the inflamed mucosa through down-regulation of the monocarboxylate transporter 1 (Thibault et al., 2007). The intracellular butyrate deficiency in colonocytes may decrease its protective effects against UC. Under such conditions, intraperitoneal butyrate that reaches the colonocytes from blood at the basolateral side could be more potent in curing UC. This could in part explain our findings that intraperitoneal butyrate was the most potent route in reducing the severity of AA colitis.

The benefits of systemic administration of butyrate could stem from the ability of butyrate to suppress adverse systemic changes occurring during UC that are pivotal to development of the disease. These systemic effects occur even in topically induced colitis models. Recently, Hou et al. (2014) demonstrated that intrarectal AA increases blood lymphocyte counts, creatinine, prostaglandin E2, and malondialdehyde concentrations, and diamine oxidase and inducible nitric oxide synthase (iNOS) activities, and decreases insulin concentrations and glutathione peroxidase activity in a porcine model of colitis. These effects were attenuated by oral administration of tributyrin, a good dietary source of butyrate and led to protection against AA colitis. These observations clearly indicate that UC patients could benefit from approaches that abrogate systemic events pivotal to the development of the disease. There is another advantage in employing systemic administration of butyrate. Patients with IBD have increased production of inflammatory cytokines like IL-12/23p40 and tumour necrosis factor- $\alpha$ (TNF- $\alpha$ ) from peripheral blood mononuclear cells. To inhibit the release of these cytokines, higher concentrations of topical butyrate are needed in these patients than in healthy subjects. This is because of impaired sensitivity to the inhibitory action of butyrate in IBD (Kovarik et al., 2011). This insensitivity could be overcome by systemic butyrate. It can be suggested from our findings that the potency of oral and intraperitoneal butyrate could be due to the ability of butyrate to prevent the systemic adverse effects of intrarectal AA. The observed differences in the potency between intraperitoneal and oral butyrate could be due to the variations in uptake and the rate of absorption between the two routes.

AA induces UC through induction of oxidative and inflammatory responses. Oxidative responses include production of oxidative enzymes like superoxide dismutase and glutathione whereas inflammatory responses are manifested by production of 
inflammatory mediators like TNF- $\alpha$, IL- $1 \beta$, IL-6, IL-8, iNOS, cyclo-oxygenase-2, myeloperoxidase, and lactate dehydrogenase (Sakthivel and Chandrasekaran, 2014; Vinod and Guruvayoorappan, 2014). Most agents that have shown potency in reducing the AA colitis lesions and signs have antioxidant and/or antiinflammatory effects (Sotnikova et al., 2013; Minaiyan et al., 2014; Sakthivel and Chandrasekaran, 2014; Vinod and Guruvayoorappan, 2014; Zhao et al., 2014). The suppressive effect of butyrate on AA induced UC clinical signs and lesions observed in our study could be mediated via the butyrate's antiinflammatory and antioxidant effects. We have demonstrated previously that butyrate modulates IL-8 production to prevent inflammatory responses (Malago et al., 2005). Several other researchers have also reported the anti-inflammatory role of butyrate in UC. Zimmerman et al. (2012) observed that butyrate inhibits interferon- $\gamma$ (IFN- $\gamma$ ) activation resulting in inhibition of iNOS up-regulation in colonocytes to suppress colonic inflammation. Butyrate has also been shown to modulate the function of intestinal macrophages, which are the most abundant immune cell type in the lamina propria. Treatment of these macrophages with butyrate leads to down-regulation of proinflammatory mediators, including nitric oxide, IL-6, and IL-12 (Chang et al., 2014). The antioxidative role of butyrate is exemplified by its ability to reduce the activity of pro-oxidative malondialdehyde and increase activities of antioxidant glutathione peroxidase, superoxide dismutase, and catalase in the colonic mucosa. It does this partly by up-regulating the gene expressions of glutathione peroxidase and catalase. In addition, butyrate promotes antioxidative status in the blood by elevating the $\alpha$-tocopherol level (Wu and Chen, 2011).

\section{Conclusions}

The results presented here provide knowledge on the potential of systemic butyrate to prevent UC lesions. This potential is higher than that of the popularly used topical butyrate and could be mediated via the ability of butyrate to suppress the inflammatory and oxidative systemic effects that are central to the development of UC. Considering this potential and the challenges in treating $\mathrm{UC}$, further research is needed to explore the exact mechanisms of systemic butyrate and whether this approach would benefit UC patients equally or more than the topically administered butyrate. Findings from such research could provide a milestone towards treating UC.

\section{Compliance with ethics guidelines}

Joshua J. MALAGO and Catherine L. SANGU declare that they have no conflict of interest.

All institutional and national guidelines for the care and use of laboratory animals were followed.

\section{References}

Bloemen, J.G., Schreinemacher, M.H., de Bruine, A.P., et al., 2010. Butyrate enemas improve intestinal anastomotic strength in rat model. Dis. Colon Rectum., 53(7):10691075. [doi:10.1007/DCR.0b013e3181d881b7]

Cetinkaya, A., Bulbuloglu, E., Kurutas, E.B., et al., 2005. Beneficial effects of $N$-acetylcysteine on acetic acidinduced colitis in rats. Tohoku J. Exp. Med., 206(2): 131-139. [doi:10.1620/tjem.206.131]

Chang, P.V., Hao, L., Offermanns, S., et al., 2014. The microbial metabolite butyrate regulates intestinal macrophage function via histone deacetylase inhibition. PNAS, 111(6):2247-2252. [doi:10.1073/pnas.1322269111]

Clark, A.J., 1921. Absorption from the peritoneal cavity. $J$. Pharmacol. Exp. Ther., 16(6):415-433.

Cummings, J.H., Pomare, E.W., Branch, W.J., et al., 1987. Short chain fatty acids in human large intestine, portal, hepatic and venous blood. Gut, 28(10):1221-1227. [doi:10.1136/gut.28.10.1221]

Dabard, J., Hudault, S., Saby, M.A., 1987. Production of butyric acid in human premature baby suffering from necrotizing enterocolitis. Proceedings of the 9th International Symposium on Gnotobiology. Versailles, p.90-95.

de Preter, V., Geboes, K.P., Bulteel, V., et al., 2011. Kinetics of butyrate metabolism in the normal colon and in ulcerative colitis: the effects of substrate concentration and carnitine on the $\beta$-oxidation pathway. Aliment. Pharmacol. Ther., 34(5):526-532. [doi:10.1111/j.1365-2036.2011. 04757.x]

de Preter, V., Arijs, I., Windey, K., et al., 2012. Impaired butyrate oxidation in ulcerative colitis is due to decreased butyrate uptake and a defect in the oxidation pathway. Inflamm. Bowel Dis., 18(6):1127-1136. [doi:10.1002/ibd. 21894]

Eeckhaut, V., Machiels, K., Perrier, C., et al., 2013. Butyricicoccus pullicaecorum in inflammatory bowel disease. Gut, 62(12):1745-1752. [doi:10.1136/gutjnl-2012-303611]

Fabia, R., Willén, R., Ar'Rajab, A., et al., 1992. Acetic acidinduced colitis in the rat: a reproducible experimental model for acute ulcerative colitis. Eur. Surg. Res., 24(4): 211-225. [doi:10.1159/000129209]

Fernández-Bañares, F., Hinojosa, J., Sánchez-Lombraña, J.L., et al., 1999. Randomized clinical trial of Plantago ovata 
seeds (dietary fiber) as compared with mesalamine in maintaining remission in ulcerative colitis. Spanish Group for the Study of Crohn's Disease and Ulcerative Colitis (GETECCU). Am. J. Gastroenterol., 94(2):427433. [doi:10.1016/S0002-9270(98)00753-9]

Ferrante, R.J., Kubilus, J.K., Lee, J., et al., 2003. Histone deacetylase inhibition by sodium butyrate chemotherapy ameliorates the neurodegenerative phenotype in Huntington's disease mice. J. Neurosci., 23(28):9418-9427.

Fusunyan, R.D., Quinn, J.J., Ohno, Y., et al., 1998. Butyrate enhances interleukin (IL)-8 secretion by intestinal epithelial cells in response to IL-1 $\beta$ and lipopolysaccharide. Pediatr. Res., 43(1):84-90. [doi:10.1203/00006450-1998 01000-00013]

Fusunyan, R.D., Quinn, J.J., Fujimoto, M., et al., 1999. Butyrate switches the pattern of chemokine secretion by intestinal epithelial cells through histone acetylation. Mol. Med., 5(9):631-640.

Hamer, H.M., Jonkers, D.M., Renes, I.B., et al., 2010a. Butyrate enemas do not affect human colonic MUC2 and TFF3 expression. Eur. J. Gastroenterol. Hepatol., 22(9): 1134-1140. [doi:10.1097/MEG.0b013e32833a6ca0]

Hamer, H.M., Jonkers, D.M., Vanhoutvin, S.A., et al., 2010b. Effect of butyrate enemas on inflammation and antioxidant status in the colonic mucosa of patients with ulcerative colitis in remission. Clin. Nutr., 29(6):738-744. [doi:10.1016/j.clnu.2010.04.002]

Harig, J.M., Soergel, K.H., Komorowski, R.A., et al., 1989. Treatment of diversion colitis with short chain fatty acid irrigation. N. Engl. J. Med., 320(1):23-28. [doi:10.1056/ NEJM198901053200105]

Holtug, K., Hove, H., Mortensen, P.B., 1995. Stimulation of butyrate absorption in the human rectum in vivo. Scand.J. Gastroenterol., 30(10):982-988. [doi:10.3109/00365529 509096342]

Hou, Y., Wang, L., Yi, D., et al., 2014. Dietary supplementation with tributyrin alleviates intestinal injury in piglets challenged with intrarectal administration of acetic acid. Br. J. Nutr., 111(10):1748-1758. [doi:10.1017/S0007114 514000038]

Hove, H., Holtug, K., Jeppesen, P.B., et al., 1995. Butyrate absorption and lactate secretion in ulcerative colitis. Dis. Colon Rectum, 38(5):519-525. [doi:10.1007/BF02148853]

Huang, N., Katz, J.P., Martin, D.R., et al., 1997. Inhibition of IL-8 gene expression in Caco-2 cells by compounds which induce histone hyperacetylation. Cytokine, 9(1): 27-36. [doi:10.1006/cyto.1996.0132]

Hudcovic, T., Kolinska, J., Klepetar, J., et al., 2012. Protective effect of Clostridium tyrobutyricum in acute dextran sodium sulphate-induced colitis: differential regulation of tumour necrosis factor- $\alpha$ and interleukin-18 in BALB/c and severe combined immunodeficiency mice. Clin. Exp. Immunol., 167(2):356-365. [doi:10.1111/j.1365-2249.2011. 04498.x]

Ke, X., Chen, J., Zhang, X., et al., 2013. Qing Hua Chang Yin attenuates lipopolysaccharide-induced inflammatory response in human intestinal cells by inhibiting NF- $\kappa \mathrm{B}$ activation. Exp. Ther. Med., 6(1):189-193.

Kovarik, J.J., Tillinger, W., Hofer, J., et al., 2011. Impaired anti-inflammatory efficacy of $n$-butyrate in patients with IBD. Eur. J. Clin. Invest., 41(3):291-298. [doi:10.1111/ j.1365-2362.2010.02407.x]

La, J.H., Kim, T.W., Sung, T.S., et al., 2003. Visceral hypersensitivity and altered colonic motility after subsidence of inflammation in a rat model of colitis. World J. Gastroenterol., 9(12):2791-2795.

Liu, H., Patel, N.R., Walter, L., et al., 2013. Constitutive expression of MMP9 in intestinal epithelium worsens murine acute colitis and is associated with increased levels of proinflammatory cytokine Kc. Am. J. Physiol. Gastrointest. Liver Physiol., 304(9):G793-G803. [doi:10. 1152/ajpgi.00249.2012]

Malago, J.J., Nondoli, H., 2008. Sodium arsenite reduces severity of dextran sulfate sodium-induced ulcerative colitis in rats. J. Zhejiang Univ.-Sci. B, 9(4):341-350. [doi:10.1631/jzus.B0720198]

Malago, J.J., Koninkx, J.F.J.G., van Dijk, J.E., 2002. The heat shock response and cytoprotection of the intestinal epithelium. Cell Stress. Chaperones, 7(2):191-199. [doi:10. 1379/1466-1268(2002)007<0191:THSRAC $>2.0 . C O ; 2]$

Malago, J.J., Koninkx, J.F.J.G., Tooten, P.C.J., et al., 2005. Anti-inflammatory properties of heat shock protein 70 and butyrate on Salmonella-induced interleukin-8 secretion in enterocyte-like Caco-2 cells. Clin. Exp. Immun., 141(1):62-71. [doi:10.1111/j.1365-2249.2005.02810.x]

Mathew, A.J., Wann, V.C., Abraham, D.T., et al., 2010. The effect of butyrate on the healing of colonic anastomoses in rats. J. Invest. Surg., 23(2):101-104. [doi:10.3109/ 08941930903469367]

Mikhailova, T.L., Sishkova, E., Poniewierka, E., et al., 2011. Randomised clinical trial: the efficacy and safety of propionyl-L-carnitine therapy in patients with ulcerative colitis receiving stable oral treatment. Aliment. Pharmacol. Ther., 34(9):1088-1097. [doi:1.1111/j.1365-2036. 2011.04844.x]

Miller, A.A., Kurschel, E., Osieka, R., et al., 1987. Clinical pharmacology of sodium butyrate in patients with acute leukemia. Eur. J. Cancer Clin. Oncol., 23(9):1283-1287. [doi:10.1016/0277-5379(87)90109-X]

Minaiyan, M., Ghassemi-Dehkordi, N., Mahzouni, P., et al., 2014. Anti-inflammatory effect of Helichrysum oligocephalum DC extract on acetic acid-induced acute colitis in rats. Adv. Biomed. Res., 3(1):87. [doi:10.4103/ 2277-9175.128000]

O'Morain, C., Segal, A.W., Levi, A.J., 1984. Elemental diet as primary treatment of acute Crohn's disease: a controlled trial. Br. Med. J. (Clin. Res. Ed.), 288(6434):1859-1862. [doi:10.1136/bmj.288.6434.1859]

Randhawa, P.K., Singh, K., Singh, N., et al., 2014. A review on chemical-induced inflammatory bowel disease models in rodents. Korean J. Physiol. Pharmacol., 18(4):279-288. [doi:10.4196/kjpp.2014.18.4.279]

Reolon, G.K., Maurmann, N., Werenicz, A., et al., 2011. Posttraining systemic administration of the histone deacetylase inhibitor sodium butyrate ameliorates agingrelated memory decline in rats. Behav. Brain Res., 221(1): 329-332. [doi:10.1016/j.bbr.2011.03.033]

Roediger, W.E.W., 1980. The colonic epithelium in ulcerative 
colitis: an energy deficiency disease. Lancet, 2(8197): 712-715. [doi:10.1016/S0140-6736(80)91934-0]

Sakthivel, K.M., Chandrasekaran, G., 2014. Protective effect of Acacia ferruginea against ulcerative colitis via modulating inflammatory mediators, cytokine profile and NF-kb signal transduction pathways. J. Environ. Pathol. Toxicol. Oncol., 33(2):83-98. [doi:10.1615/JEnviron PatholToxicolOncol.2014008425]

Sanderson, I.R., 1997. Diet and gut inflammation. Curr. Opin. Gastroenterol., 13(6):518-524. [doi:10.1097/00001574199711000-00013]

Sauer, J., Richter, K.K., Pool-Zobel, B.L., 2007. Physiological concentrations of butyrate favorably modulate genes of oxidative and metabolic stress in primary human colon cells. J. Nutr. Biochem., 18(11):736-745. [doi:10.1016/j. jnutbio.2006.12.012]

Scheppach, W., Sommer, H., Kirchner, T., et al., 1992. Effect of butyrate enemas on the colonic mucosa in distal ulcerative colitis. Gastroenterology, 103(1):51-56.

Silingardi, D., Scali, M., Belluomini, G., et al., 2010. Epigenetic treatments of adult rats promote recovery from visual acuity deficits induced by long-term monocular deprivation. Eur. J. Neurosci., 31(12):2185-2192. [doi:10. $1111 / \mathrm{j} .1460-9568.2010 .07261 . \mathrm{x}]$

Sotnikova, R., Nosalova, V., Navarova, J., 2013. Efficacy of quercetin derivatives in prevention of ulcerative colitis in rats. Interdiscip. Toxicol., 6(1):9-12. [doi:10.2478/intox2013-0002]

Sun, X., Zhang, B., Hong, X., et al., 2013. Histone deacetylase inhibitor, sodium butyrate, attenuates gentamicin-induced nephrotoxicity by increasing prohibitin protein expression in rats. Eur. J. Pharmacol., 707(1-3):147-154. [doi:10.1016/j.ejphar.2013.03.018]

Thibault, R., de Coppet, P., Daly, K., et al., 2007. Downregulation of the monocarboxylate transporter 1 is involved in butyrate deficiency during intestinal inflammation. Gastroenterology, 133(6):1916-1927. [doi:10. 1053/j.gastro.2007.08.041]

Thibault, R., Blachier, F., Darcy-Vrillon, B., et al., 2010. Butyrate utilization by the colonic mucosa in inflammatory bowel diseases: a transport deficiency. Inflamm. Bowel Dis., 16(4):684-965. [doi:10.1002/ibd.21108]

Torpy, J.M., Lynm, C., Golub, R.M., 2012. Ulcerative colitis. JAMA, 307(1):104. [doi:10.1001/jama.2011.1889]

Treem, W.R., Ahsan, N., Shoup, M., et al., 1994. Fecal short-chain fatty acids in children with inflammatory bowel disease. J. Pediatr. Gastroenterol. Nutr., 18(2): 159-164. [doi:10.1097/00005176-199402000-00007]

Venkatraman, A., Ramakrishna, B.S., Shaji, R.V., et al., 2003. Amelioration of dextran sulfate colitis by butyrate: role of heat shock protein 70 and NF-кB. Am. J. Physiol. Gastrointest. Liver Physiol., 285(1):G177-G184. [doi:10. 1152/ajpgi.00307.2002]

Vernia, P., Monteleone, G., Grandinetti, G., et al., 2000. Combined oral sodium butyrate and mesalazine treatment compared to oral mesalazine alone in ulcerative colitis: randomized, double-blind, placebo-controlled pilot study. Dig. Dis. Sci., 45(5):976-981. [doi:10.1023/A:100553741
1244]

Vieira, E.L., Leonel, A.J., Sad, A.P., et al., 2012. Oral administration of sodium butyrate attenuates inflammation and mucosal lesion in experimental acute ulcerative colitis. $J$. Nutr. Biochem., 23(5):430-436. [doi:10.1016/j.jnutbio. 2011.01.007]

Vinod, P.V., Guruvayoorappan, C., 2014. Protective effect of marine mangrove Rhizophora apiculata on acetic acid induced experimental colitis by regulating anti-oxidant enzymes, inflammatory mediators and nuclear factorkappa B subunits. Int. Immunopharmacol., 18(1):124134. [doi:10.1016/j.intimp.2013.11.007]

Wu, G.D., Huang, N., Wen, X., et al., 1999. High-level expression of IKB- $\beta$ in the surface epithelium of the colon: in vitro evidence for an immunomodulatory role. $J$. Leukoc. Biol., 66(6):1049-1056.

Wu, W.T., Chen, H.L., 2011. Konjac glucomannan and inulin systematically modulate antioxidant defense in rats fed a high-fat fiber-free diet. J. Agric. Food Chem., 59(17): 9194-9200. [doi:10.1021/jf202060p]

Zhao, L., Wu, H., Zhao, A., et al., 2014. The in vivo and in vitro study of polysaccharides from a two-herb formula on ulcerative colitis and potential mechanism of action. $J$. Ethnopharmacol., 153(1):151-159. [doi:10.1016/j.jep. 2014.02.008]

Zhong, T., Qing, Q.J., Yang, Y., et al., 2014. Repression of contexual fear memory induced by isoflurane is accompanied by reduction in histone acetylation and rescued by sodium butyrate. Br. J. Anaesth., 113(4):634-643. [doi:10. 1093/bja/aeu184]

Zimmerman, M.A., Singh, N., Martin, P.M., et al., 2012. Butyrate suppresses colonic inflammation through HDAC1dependent Fas upregulation and Fas-mediated apoptosis of T cells. Am. J. Physiol. Gastrointest. Liver Physiol., 302(12):G1405-G1415. [doi:10.1152/ajpgi.00543.2011]

\section{中文概要}

题 目: 腹腔注射丁酸盐对大鼠乙酸性结肠炎的预防作用

目 的: 探索腹腔注射丁酸盐对防止乙酸性结肠炎的疗效。

创新点: 首次对大鼠进行腹腔注射丁酸盐, 通过与直肠灌 注和口服比较, 探索三种不同给药方式对预防乙 酸性结肠炎的疗效差异。

方 法: 以 40 只 Wistar 大鼠为实验对象, 分组进行连续 7 天的腹腔注射、直肠灌注和口服 $100 \mathrm{mg} / \mathrm{kg}$ 丁酸 钠 $(\mathrm{SB})$ ，第 8 天进行乙酸 $(\mathrm{AA})$ 直肠灌注, 48 小时后处死。记录实验大鼠的临床症状, 包括 体重减少、腹泻、便血等。对结肠切片进行组织 病理学观察, 最后对试验数据进行统计分析。

结 论: 腹腔注射、直肠灌注和口服丁酸盐均能明显缓解 大鼠乙酸性结肠炎的炎症, 其中以腹腔注射疗效 最佳。

关键词: 丁酸盐; 口服; 腹腔注射; 直肠灌注; 乙酸; 溃 疡性结肠炎 